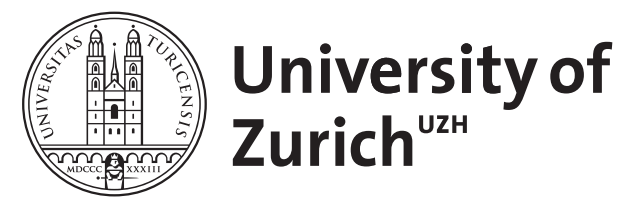

\title{
Regulation of dolichol-linked glycosylation
}

Welti, Michael

\begin{abstract}
In the majority of congenital disorders of glycosylation, the assembly of the glycan precursor GlcNAc2Man9Glc3 on the polyprenol carrier dolichyl-pyrophosphate is compromised. Because N-linked glycosylation is essential to life, most types of congenital disorders of glycosylation represent partial losses of enzymatic activity. Consequently, increased availability of substrates along the glycosylation pathway can be beneficial to increase product formation by the compromised enzymes. Recently, we showed that increased dolichol availability and improved N-linked glycosylation can be achieved by inhibition of squalene biosynthesis. This review summarizes the current knowledge on the biosynthesis of dolichol-linked glycans with respect to deficiencies in N-linked glycosylation. Additionally, perspectives on therapeutic treatments targeting dolichol and dolichol-linked glycan biosynthesis are examined
\end{abstract}

DOI: https://doi.org/10.1007/s10719-012-9417-y

Posted at the Zurich Open Repository and Archive, University of Zurich ZORA URL: https://doi.org/10.5167/uzh-156204

Journal Article

Published Version

Originally published at:

Welti, Michael (2013). Regulation of dolichol-linked glycosylation. Glycoconjugate journal, 30(1):51-56. DOI: https://doi.org/10.1007/s10719-012-9417-y 


\title{
Regulation of dolichol-linked glycosylation
}

\author{
Michael Welti
}

Received: 1 March 2012 /Revised: 6 June 2012 / Accepted: 11 June 2012 / Published online: 21 June 2012

(C) Springer Science+Business Media, LLC 2012

\begin{abstract}
In the majority of congenital disorders of glycosylation, the assembly of the glycan precursor $\mathrm{GlcNAc}_{2-}$ $\mathrm{Man}_{9} \mathrm{Glc}_{3}$ on the polyprenol carrier dolichyl-pyrophosphate is compromised. Because N-linked glycosylation is essential to life, most types of congenital disorders of glycosylation represent partial losses of enzymatic activity. Consequently, increased availability of substrates along the glycosylation pathway can be beneficial to increase product formation by the compromised enzymes. Recently, we showed that increased dolichol availability and improved N-linked glycosylation can be achieved by inhibition of squalene biosynthesis. This review summarizes the current knowledge on the biosynthesis of dolichol-linked glycans with respect to deficiencies in N-linked glycosylation. Additionally, perspectives on therapeutic treatments targeting dolichol and dolichol-linked glycan biosynthesis are examined.
\end{abstract}

Keywords CDG $\cdot$ Dolichol $\cdot$ Mevalonate pathway $\cdot$ Statins

\section{Dolichol biosynthesis and its role in N-linked glycosylation}

$\mathrm{N}$-linked glycosylation occurs in all domains of life and the same basic molecular principles underlie this type of posttranslational modification [1,2]. Briefly, an oligosaccharide precursor is assembled on a lipid carrier before the oligosaccharide is transferred to a target protein. In eukaryotes, N-linked protein glycosylation requires the assembly of the oligosaccharide precursor at both the outer and inner leaflet of the ER

\section{Welti $(\bowtie)$}

Institute of Physiology, University of Zürich,

Winterthurerstrasse 190,

8057 Zürich, Switzerland

e-mail: michael.welti@uzh.ch membrane [3]. The precursor is first assembled on dolichylpyrophosphate (Dol-PP) starting at the cytoplasmic side. The intermediate Dol-PP-GlcNAc $\mathrm{Man}_{5}$ is then flipped to the luminal side of the ER where the assembly proceeds to Dol-PP$\mathrm{GlcNAc}_{2} \mathrm{Man}_{9} \mathrm{Glc}_{3}$. Note worthily, dolichol (Dol) serves as lipid carrier in eukaryotes and archaea whereas another isoprenoid alcohol, bactoprenol, is used in bacteria. Apart from its role as carrier of the oligosaccharide $\mathrm{GlCNAc}_{2} \mathrm{Man}_{9} \mathrm{Glc}_{3}$, Dol is used to form the activated monosaccharides Dol-P-Man and Dol-P-Glc, which are substrates for glycosyltransferases involved in Nglycosylation, O-mannosylation, C-mannosylation and glycosylphosphatidylinositol (GPI) anchor biosynthesis.

Dol biosynthesis begins at the mevalonate pathway (Fig. 1) $[4,5]$, which produces essential isoprenoids. Isoprenoids are divided into two classes: sterol isoprenoids, such as cholesterol and steroid hormones, and non-sterol isoprenoids, encompassing polyprenols, ubiquinone, and Dol. Starting from acetylCoA via acetoacetyl-CoA, 3-hydroxy-3-methylglutaryl-CoA (HMG-CoA) is first produced. The reduction from HMG$\mathrm{CoA}$ to mevalonate is catalyzed by the rate-limiting HMGCoA reductase (HMGCR) enzyme. Mevalonate is then phosphorylated to mevalonate-P by mevalonate kinase (MVK). A second phosphorylation step and subsequent decarboxylation leads to isopentenyl-5-pyrophosphate (IPP), which represents an important building block for isoprenoids. IPP is an activated isoprene unit of five carbon atoms and is used to form farnesylpyrophosphate. At this point, the pathway diverges to feed on the one hand the biosynthesis of cholesterol and on the other hand the biosynthesis of non-sterol isoprenoids. To obtain Dol, dehydrodolichyl diphosphate synthase (DHDDS) catalyzes the stepwise head-to-tail cis addition of IPP to farnesylpyrophosphate, thus giving rise to polyprenyl-pyrophosphates of 15 to 19 isoprene units. These polyprenyl-pyrophosphates are then dephosphorylated by pyro- or monophosphatases. The resulting polyprenols are reduced at the $\alpha 1$ position to become 
Dol of different lengths. Dol is then phosphorylated by Dol kinase (DOLK). Dol-P can then be utilized as the carrier for the oligosaccharide $\mathrm{GlcNAc}_{2} \mathrm{Man}_{9} \mathrm{Glc}_{3}$ or as a carrier for Man and Glc. Dol-P-Man is produced by the Dol-P-Man synthase (DPM1-3) that transfers Man from GDP-Man to Dol-P [6]. Similarly, Dol-P-Glc is produced by the glucosyltransferase ALG5 that transfers Glc from UDP-Glc to Dol-P [7].

Importantly, Dol levels at the cytoplasmic leaflet of the ER are not only maintained by de novo synthesis but also depend on recycling of discharged Dol-PP and Dol-P. Once the oligosaccharide precursor is transferred to a target protein, DolPP is released and dephosphorylated to Dol-P by the luminal phosphatase DOLPP1 [8]. Dol-P is then flipped across the membrane to the cytoplasmic leaflet by a yet unknown mechanism [9]. Recycling of Dol-P and Dol-PP contributes significantly to the Dol pool available glycosylation in the ER. Accordingly, a defect of the DOLPP1 orthologue in yeast leads to impaired N-linked glycosylation [10].

\section{Deficiency of dolichol biosynthesis—a new family of CDG}

Several forms of congenital disorders of glycosylation (CDG) caused by Dol biosynthesis defects have been characterized recently. The long sought polyprenol reductase has been identified though the description of SRD5A3-CDG [11] and DHDDS deficiency was discovered in a subgroup of retinitis pigmentosa patients [12]. Moreover, the clinical picture of Dol kinase deficiency was extended through the description of new cases presenting with dilated cardiomyopathy [13].

Proximal to Dol, a defect along the mevalonate pathways has also been linked to diseases. Mutations of MVK (Fig. 1) cause of two different forms: mevalonic aciduria (MVA) [14] and hyperimmunoglobulinemia D syndrome (HIDS) [15]. In MVA, MVK activity is reduced to $0-4 \%$ of normal values leading to death in early childhood. Typcial symptoms (Table 1) are psychomotor retardation, failure to thrive, progressive cerebellar ataxia, dysmorphic features, progressive visual impairment and frequent febrile attacks $[5,16$, 17]. Psychomotor retardation, failure to thrive, dysmorphic features, and visual impairment are also frequently found in $\mathrm{CDG}$, thus suggesting possible glycosylation problems in MVA. HIDS is a milder form of the disease correlating with residual MVK activity of 5-15\% of normal values. In HIDS, febrile attacks and skin rashes start in early childhood and can be triggered by diverse events, such as vaccinations or minor infections. The febrile disposition is believed to be linked to insufficient levels of anti-inflammatory isoprenylated proteins. Low geranylgeranyl-PP in HIDS leads to
Fig. 1 Dolichol and cholesterol biosynthesis pathway. Two acetyl building blocks are required for $\mathrm{HMG}-\mathrm{CoA}$ formation. HMG-CoA reductase (HMGCR) catalyzes the rate limiting step, the formation of mevalonate. Mevalonate kinase (MVK) phosphorylates mevalonate in a CTP-dependent manner. Sequential action of dehydrodolichyl diphosphate synthase (DHDDS), pyro- or monophophatases, $5 \alpha$ steroid reductase type 3 (SRD5A3), and dolichol kinase (DOLK) produce Dol-P. Dol-P-Man synthase (DPM1-3) transfers a mannose to Dol-P. Squalene synthase (FDFT1) produces squalene from farnesyl-PP and subsequent action of squalene epoxidase (SQLE), and 2,3oxidosqualene cyclase (OSC) leads to lanosterol. Dotted arrows indicate simplifications of the biosynthetic pathway and * point out known congenital defects in dolichol biosynthesis of the respective enzymes

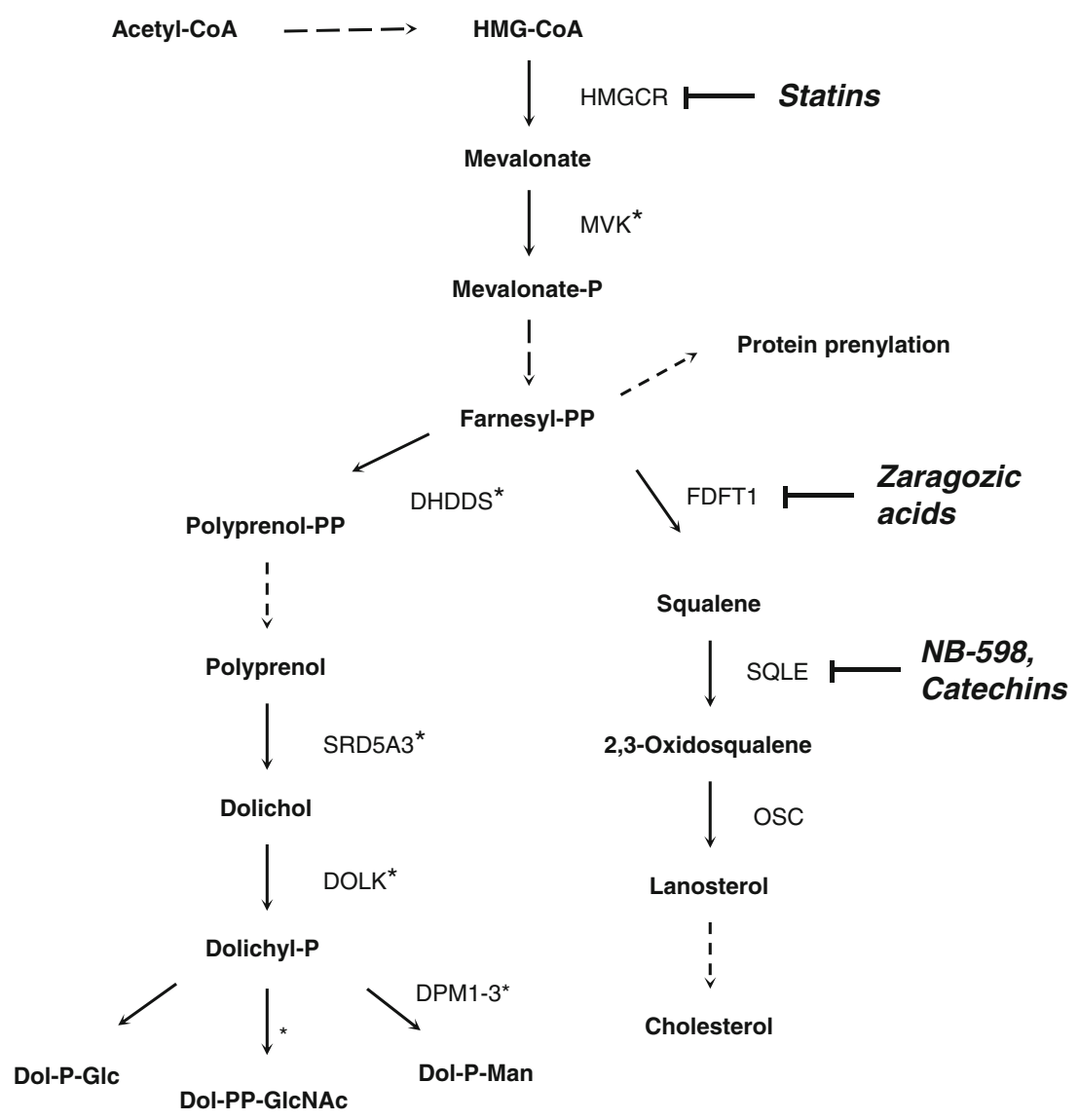


Table 1 Disorders of dolichol biosynthesis and their respective symptoms

Symptoms are distinguished between $* *$ for typical/dominant symptoms and $*$ for sporadic symptoms or symptoms of moderate severity

${ }^{\mathrm{a}}$ Classical DOLK-CDG, ${ }^{\mathrm{b}}$ DOLK defect in dilated cardiomyopathy

\begin{tabular}{|c|c|c|c|c|c|c|c|c|}
\hline & MVA & HIDS & $\begin{array}{l}\text { DHDDS- } \\
\text { CDG }\end{array}$ & $\begin{array}{l}\text { SRD5A3- } \\
\text { CDG }\end{array}$ & $\begin{array}{l}\text { DOLK- } \\
\text { CDG }^{\mathrm{a}}\end{array}$ & $\begin{array}{l}\text { DOLK- } \\
\text { CDG }^{\mathrm{b}}\end{array}$ & $\begin{array}{l}\text { DPM1- } \\
\text { CDG }\end{array}$ & $\begin{array}{l}\text { DPM3- } \\
\text { CDG }\end{array}$ \\
\hline Cardiomyopathy & & & & * & $* *$ & $* *$ & & $*$ \\
\hline Cerebellar ataxia / malformation & $* *$ & $* *$ & & $* *$ & & & $* *$ & \\
\hline Coagulopathy & & & & $* *$ & & & $* *$ & \\
\hline Dysmorphic features & $* *$ & & & $* *$ & $* *$ & & & \\
\hline Failure to thrive & $* *$ & & & & & & & \\
\hline Febrile attacks & $* *$ & $* *$ & & & & & & \\
\hline Hypoglycemia & & & & & $* *$ & & & \\
\hline Hypotonia & & & & & $* *$ & & $* *$ & \\
\hline Microcephaly & $* *$ & $*$ & & & $* *$ & & $* *$ & \\
\hline Muscular dystrophy & & & & & & & $* *$ & $*$ \\
\hline Myopathy & & & & & & & $* *$ & $*$ \\
\hline Ocular malfunctions & $* *$ & & $* *$ & $* *$ & $* *$ & & $* *$ & \\
\hline Psychomotor retardation & $* *$ & $* *$ & & $* *$ & $* *$ & $*$ & $* *$ & \\
\hline Seizures & $* *$ & $*$ & & & $* *$ & & $* *$ & \\
\hline Skin disorders & $* *$ & $* *$ & & $*$ & $* *$ & $*$ & & \\
\hline
\end{tabular}

caspase-1 activation and IL-1 $\beta$ secretion $[18,19]$. Other symptoms found in HIDS (Table 1) are mental retardation, ataxia, ocular symptoms and epilepsy [17]. Diagnosing HIDS involves the detection of increased mevalonic acid in urine and increased serum immunoglobulin D and A. Apart from febrile attacks, the clinical features of MVA and HIDS are reminiscent of CDG. Indeed, metabolic labelling experiments with $\left[{ }^{14} \mathrm{C}\right]$ galactose in MVK deficient fibroblasts revealed lower secretion of the radio-labelled macromolecules indicating impaired glycosylation, which is possibly due to decreased formation of Dol and Dol-P [20]. However, systematic analysis of glycosylation in HIDS has not been performed yet.

Recently, DHDDS deficiency (Fig. 1) has been linked to inherited retinitis pigmentosa [12], a disorder causing retinal degeneration with an estimated incidence of 1 in 3,000 4,500 . The single mutation identified was present in a very small subgroup of retinitis pigmentosa patients [21]. Interestingly, DHDDS patients did not show typical CDG symptoms despite the central role of DHDDS in Dol production (Table 1). This fact suggests that DHDDS mutations associated with retinitis pigmentosa only partially impair polyprenol-PP formation and that only few photoreceptorspecific proteins may be sensitive to a reduced polyprenolPP pool. Note worthily, inhibition of N-linked glycosylation by tunicamycin also leads to retinal degeneration in Xenopus $[22,23]$. Retinitis pimentosa is also associated with MVK deficiency and with cases of phosphomannomutase 2 deficiency in PMM2-CDG [24].

The third disorder of Dol biosynthesis is a recently described type of CDG, in which the SRD5A3 gene (Fig. 1) is affected $[25,26]$. SRD $5 A 3$ encodes the steroid $5 \alpha$ reductase type 3 protein, which reduces the $\alpha$-isoprene in polyprenol to form Dol. Symptoms of SRD5A3-CDG are typical for glycosylation disorders and encompass ocular malformations, cerebellar vermis hypoplasia, skin lesions, psychomotor retardation, and facial dysmorphism (Table 1). Strikingly, these multisystemic manifestations strongly differ from those found in DHDDS-CDG despite the proximity of the two enzymes in the Dol pathway. Loss of whole Nglycan chains on proteins has been described in SRD5A3CDG. Different mutations of SRD5A3 lead to truncated forms of the protein resulting in loss of function. Residual levels of Dol in SRD5A3 deficient cells suggest an alternative pathway for Dol biosynthesis.

Dol kinase deficiency, characterizing DOLK-CDG, impairs the phosphorylation of Dol to Dol-P (Fig. 1). DOLK-CDG presents with severe phenotypes including hypotonia, skin disorders, and the loss of hair [27]. Moreover, cardiomyopathy, seizures, hypoglycemia, microcephaly, and visual impairment can occur as well (Table 1). Due to the severity of the symptoms, most $D O L K$ mutations generally cause a severe disease associated with infant lethality. Reduced DOLK activity decreases the availability of Dol-P and thus impairs the assembly Dol-PP-GlcNAc ${ }_{2} \mathrm{Man}_{9} \mathrm{Glc}_{3}$ and N-linked glycosylation. Recently, 11 children with dilated cardiomyopathy (DCM), a disease possibly linked to sudden cardiac death and heart failure, were found to have a novel mutation in DOLK [13]. Besides DCM, only few patients exhibit additional symptoms like ichtyosis, failure to thrive, and mild neurological involvement. Biochemical analysis of Dol-P dependent glycosylation pathways of the DCM heart tissue indicated reduced Omannosylation of $\alpha$-dystroglycan [28]. Remarkably, abnormal $\mathrm{N}$-glycosylation of serum transferrin was equally pronounced 
in all forms of DOLK-CDG. The phenotypes of DCM strongly contrast with the originally described features of DOLK-CDG [27], thus suggesting a possible tissue-specific regulation of Dol-P dependent glycosylation.

Human Dol-P-Man synthase (DPM1-3, Fig. 1) consists of 3 subunits. DPM1 is the catalytic subunit and DPM2/3 are regulatory and membrane anchoring proteins residing in the ER membrane [6]. So far, two forms of Dol-P-Man synthase deficiency have been associated with mutations in the DPM1 and DPM3 genes. DPM1-CDG is characterized by recurrent seizures, hypotonia, developmental delay, dysmorphic features, microcephaly, visual impairment, and in some cases ataxia and coagulopathy (Table 1) [29-32]. Lower Dol-PMan levels lead to impaired Dol-PP-GlcNAc ${ }_{2} \mathrm{Man}_{9} \mathrm{Glc}_{3}$ assembly, abnormal N-linked protein glycosylation, and decreased formation of GPI anchored proteins. DPM3-CDG appears to be a milder form of Dol-P-Man synthase deficiency, for which only one patient has been found so far [33]. The symptoms included mild myopathy, a dilated cardiomyopathy, moderate muscular dystrophy, and a single stroke-like episode. N-glycosylation of serum transferrin was found to be only slightly abnormal in DPM3-CDG. In DPM1-CDG and DPM3-CDG, the severity seems to reflect the molecular functions of the affected subunits. A deficiency of the catalytic subunit DPM1 leads to much more severe phenotypes than a deficieny in the tethering subunit DPM3.

In general, the clinical severity of Dol-related diseases does not correlate with the relative position of the deficient enzymes along the biosynthesis pathway. Defects of Dol biosynthesis are severe since not only the assembly of Nglycosylation is impaired, but also GPI anchor formation and O-mannosylation are affected. This notion is confirmed in SRD5A3-CDG that present with severe multisystemic phenotypes. However, the discovery of DHDDS-CDG with a very specific phenotype and lack of the typical CDG symptoms suggests a complex regulation of Dol-dependent glycosylation. Future efforts addressing Dol biosynthesis will improve our comprehension of the regulation of $\mathrm{N}$ glycosylation and provide therapeutic perspectives.

\section{Therapeutics targeting dolichol}

To date, only two forms of CDG are treatable. MPI-CDG can be successfully treated by oral mannose supplementation [34, 35]. In GFTP-CDG, deficient GDP-fucose transport can be compensated by nutritional fucose supplementation [36]. The hypomorphic nature of most CDG mutations allows for a compensatory approach, for instance by providing more Dol to overcome the compromised assembly of Dol-PP$\mathrm{GlcNAc}_{2} \mathrm{Man}_{9} \mathrm{Glc}_{3}$. Along this line, inhibition of squalene synthase (FDFT1, Fig. 1) was shown to improve N-linked protein glycosylation and GPI-anchored protein expression in
DPM1-CDG fibroblasts [37]. Additional drugs such as clofibrate were also tested as a way to increase GPI anchor availability in DPM1-CDG fibroblasts. Clofibrate decreases cholesterol levels by targeting lipid metabolism via activation of peroxisome proliferator-activated receptors, especially PPAR $\alpha[38,39]$. Indeed, surface expression of the GPI anchored protein CD59 was increased upon clofibrate treatment (unpublished data). Similarly, the adenylate cyclase activator forskolin [40] increased surface expression of CD59 as well. This was in accordance with the notion that high levels of cyclic AMP lead to higher activity of DHDDS and thus increased Dol levels [41, 42].

New therapeutic possibilities arise for CDG with the advance of non-statin cholesterol lowering drugs, which target the late pathway of cholesterol biosynthesis. Presently, statins are the prevalent medication to lower cholesterol and decrease cardiovascular disease [43]. Statins are inhibitors of HMGCR (Fig. 1), the rate limiting enzyme at the beginning of the mevalonate pathway. The effect of statins on Dol levels has not been investigated systematically. Hela cells treated with pravastatin showed a decrease in Dol-P by $65 \%$ [44]. However, rats treated with lovastatin did not show a change of total Dol-P levels but increase of Dol-P in the liver [45]. The potential of different statins to upregulate Dol could be subject of further investigations.

Inhibition of the first committed enzyme of cholesterol biosynthesis, squalene synthase (FDFT1, Fig. 1), has proven effective in lowering cholesterol production [46]. Prominent among these inhibitors are zaragozic acids, which were identified in a screen of fungal compounds for cholesterol lowering activity [47]. Changes in Dol-P patterns were observed in human fibroblasts treated with zaragozic acid A. Also, DPM1-CDG fibroblasts showed improved N-linked glycosylation upon treatment with zaragozic acid A [37]. Moreover, the expression of the GPI-anchored CD59 protein could be normalized in DPM1CDG fibroblasts after treatment. Since zaragozic acid is well tolerated in animals [48, 49], this class of FDFT1 inhibitors might be considered for clinical testing in CDG.

Squalene epoxidase (SQLE) catalyzes the next committed step of cholesterol biosynthesis after FDFT1 (Fig. 1). Inhibition of SQLE by the compound NB-598 ((E) $N$-ethyl$N$-(6,6-dimethyl-2-hepten-4-ynyl)-3-[(3,3'-bith iophen-5-yl) methoxy]benzene-methanamine) successfully reduced cholesterol levels [50, 51], although no changes in Dol levels were detected in treated HepG2 cells [52]. In spite of this early finding, newly developed SQLE inhibitors should be considered as promising candidates for an upregulation of Dol in CDG cells. Moreover, green tea has been attributed a cholesterol lowering effect, which was linked to SQLE inhibition [53]. In a recent study, oral supplementation with the main green tea catechin epigallocatechin gallate was shown to lower low density lipoprotein associated cholesterol [54]. The effects of epigallocatechin gallate on Dol 
have not been tested yet. Catechins might represent another family of compounds with the potential to upregulate Dol in CDG cells by inhibition of SQLE.

In conclusion, inhibitors of late cholesterol biosynthesis have a potential increasing effect on Dol levels and should be accordingly tested in CDG cells. Much effort is invested in the development of cholesterol lowering drugs in the context of cardiovascular diseases. Therefore, potential CDG therapies could profit from the discovery of novel cholesterol lowering drugs in the future.

\section{Outlook}

The recent descriptions of tissue-restricted symptoms in DHDDS-CDG and of a novel pathology associated to DOLK deficiency suggest a pronounced tissue specificity of Dolrelated biology. Accordingly, additional diseases are likely to be associated with local alterations of Dol biosynthesis in the near future. The study of these tissue-restricted diseases represents valuable models to better understand the regulation of Dol biosynthesis and its impact on various types of glycosylation. Novel therapeutic approaches may be deduced from such insights. The treatment of CDG is still very limited and merits further attention. Interference of cholesterol production and thus upregulation of Dol biosynthesis certainly represents a valuable approach to improve glycosylation in CDG.

\section{References}

1. Schwarz, F., Aebi, M.: Mechanisms and principles of N-linked protein glycosylation. Curr. Opin. Struct. Biol. 21(5), 576-582 (2011)

2. Larkin, A., Imperiali, B.: The expanding horizons of asparaginelinked glycosylation. Biochemistry 50(21), 4411-4426 (2011)

3. Kornfeld, R., Kornfeld, S.: Assembly of asparagine-linked oligosaccharides. Annu. Rev. Biochem. 54, 631-664 (1985)

4. Swiezewska, E., Danikiewicz, W.: Polyisoprenoids: structure, biosynthesis and function. Prog. Lipid Res. 44(4), 235-258 (2005)

5. Buhaescu, I., Izzedine, H.: Mevalonate pathway: a review of clinical and therapeutical implications. Clin. Biochem. 40(9-10), 575-584 (2007)

6. Maeda, Y., Kinoshita, T.: Dolichol-phosphate mannose synthase: structure, function and regulation. Biochim. Biophys. Acta 1780 (6), 861-868 (2008)

7. Heesen, S., et al.: Isolation of the ALG5 locus encoding the UDPglucose:dolichyl-phosphate glucosyltransferase from Saccharomyces cerevisiae. Eur. J. Biochem. 224(1), 71-79 (1994)

8. Rush, J.S., et al.: Identification and characterization of a cDNA encoding a dolichyl pyrophosphate phosphatase located in the endoplasmic reticulum of mammalian cells. J. Biol. Chem. 277 (47), 45226-45234 (2002)

9. Rush, J.S., et al.: Recycling of dolichyl monophosphate to the cytoplasmic leaflet of the endoplasmic reticulum after the cleavage of dolichyl pyrophosphate on the lumenal monolayer. J. Biol. Chem. 283(7), 4087-4093 (2008)

10. van Berkel, M.A., et al.: The Saccharomyces cerevisiae CWH8 gene is required for full levels of dolichol-linked oligosaccharides in the endoplasmic reticulum and for efficient $\mathrm{N}$-glycosylation. Glycobiology 9(3), 243-253 (1999)

11. Cantagrel, V., Lefeber, D.J.: From glycosylation disorders to dolichol biosynthesis defects: a new class of metabolic diseases. J. Inherit. Metab. Dis. 34(4), 859-867 (2011)

12. Zelinger, L., et al:: A missense mutation in DHDDS, encoding dehydrodolichyl diphosphate synthase, is associated with autosomalrecessive retinitis pigmentosa in Ashkenazi Jews. Am. J. Hum. Genet. 88(2), 207-215 (2011)

13. Lefeber, D.J., et al.: Autosomal recessive dilated cardiomyopathy due to DOLK mutations results from abnormal dystroglycan Omannosylation. PLoS Genet. 7(12), e1002427 (2011)

14. Stoffels, M., Simon, A.: Hyper-IgD syndrome or mevalonate kinase deficiency. Curr. Opin. Rheumatol. 23(5), 419-423 (2011)

15. Goldfinger, S.: The inherited autoinflammatory syndrome: a decade of discovery. Trans. Am. Clin. Climatol. Assoc. 120, 413-418 (2009)

16. Drenth, J.P., Haagsma, C.J., van der Meer, J.W.: Hyperimmunoglobulinemia $\mathrm{D}$ and periodic fever syndrome. The clinical spectrum in a series of 50 patients. International Hyper-IgD Study Group. Medicine (Baltimore) 73(3), 133-144 (1994)

17. Haas, D., Hoffmann, G.F.: Mevalonate kinase deficiencies: from mevalonic aciduria to hyperimmunoglobulinemia D syndrome. Orphanet J. Rare Dis. 1, 13 (2006)

18. Mandey, S.H., et al.: A role for geranylgeranylation in interleukin1beta secretion. Arthritis Rheum. 54(11), 3690-3695 (2006)

19. Kuijk, L.M., et al.: Statin synergizes with LPS to induce IL-1beta release by THP-1 cells through activation of caspase-1. Mol. Immunol. 45(8), 2158-2165 (2008)

20. Hoffmann, G.F., et al.: Regulatory adaptation of isoprenoid biosynthesis and the LDL receptor pathway in fibroblasts from patients with mevalonate kinase deficiency. Pediatr. Res. 41(4 Pt 1), 541-546 (1997)

21. Rosenberg, T.: Epidemiology of hereditary ocular disorders. Dev. Ophthalmol. 37, 16-33 (2003)

22. Fliesler, S.J., Rapp, L.M., Hollyfield, J.G.: Photoreceptor-specific degeneration caused by tunicamycin. Nature 311(5986), 575-577 (1984)

23. Fliesler, S.J., Rayborn, M.E., Hollyfield, J.G.: Membrane morphogenesis in retinal rod outer segments: inhibition by tunicamycin. J. Cell Biol. 100(2), 574-587 (1985)

24. Grunewald, S.: The clinical spectrum of phosphomannomutase 2 deficiency (CDG-Ia). Biochim. Biophys. Acta 1792(9), 827-834 (2009)

25. Cantagrel, V., et al.: SRD5A3 is required for converting polyprenol to dolichol and is mutated in a congenital glycosylation disorder. Cell 142(2), 203-217 (2010)

26. Kasapkara, C.S., et al.: SRD5A3-CDG: A patient with a novel mutation. Eur. J. Paediatr. Neurol. (2012)

27. Kranz, C., et al.: A defect in dolichol phosphate biosynthesis causes a new inherited disorder with death in early infancy. Am. J. Hum. Genet. 80(3), 433-440 (2007)

28. Michele, D.E., et al.: Dystroglycan matrix receptor function in cardiac myocytes is important for limiting activity-induced myocardial damage. Circ. Res. 105(10), 984-993 (2009)

29. Dancourt, J., et al.: A new intronic mutation in the DPM1 gene is associated with a milder form of CDG Ie in two French siblings. Pediatr. Res. 59(6), 835-839 (2006)

30. Garcia-Silva, M.T., et al:: Congenital disorder of glycosylation (CDG) type Ie. A new patient. J. Inherit. Metab. Dis. 27(5), 591$600(2004)$

31. Imbach, T., et al.: Deficiency of dolichol-phosphate-mannose synthase-1 causes congenital disorder of glycosylation type Ie. J. Clin. Invest. 105(2), 233-239 (2000)

32. Kim, S., et al.: Dolichol phosphate mannose synthase (DPM1) mutations define congenital disorder of glycosylation Ie (CDGIe). J. Clin. Invest. 105(2), 191-198 (2000)

33. Lefeber, D.J., et al.: Deficiency of Dol-P-Man synthase subunit DPM3 bridges the congenital disorders of glycosylation with the dystroglycanopathies. Am. J. Hum. Genet. 85(1), 76-86 (2009) 
34. Niehues, R., et al.: Carbohydrate-deficient glycoprotein syndrome type Ib. Phosphomannose isomerase deficiency and mannose therapy. J. Clin. Invest. 101(7), 1414-1420 (1998)

35. Westphal, V., et al.: Genetic and metabolic analysis of the first adult with congenital disorder of glycosylation type Ib: long-term outcome and effects of mannose supplementation. Mol. Genet. Metab. 73(1), 77-85 (2001)

36. Marquardt, T., et al.: Correction of leukocyte adhesion deficiency type II with oral fucose. Blood 94(12), 3976-3985 (1999)

37. Haeuptle, M.A., et al.: Improvement of dolichol-linked oligosaccharide biosynthesis by the squalene synthase inhibitor zaragozic acid. J. Biol. Chem. 286(8), 6085-6091 (2011)

38. Forman, B.M., Chen, J., Evans, R.M.: Hypolipidemic drugs, polyunsaturated fatty acids, and eicosanoids are ligands for peroxisome proliferator-activated receptors alpha and delta. Proc. Natl. Acad. Sci. U. S. A. 94(9), 4312-4317 (1997)

39. Abourbih, S., et al.: Effect of fibrates on lipid profiles and cardiovascular outcomes: a systematic review. Am. J. Med. 122(10), 962 e1-8 (2009)

40. Insel, P.A., Ostrom, R.S.: Forskolin as a tool for examining adenylyl cyclase expression, regulation, and $\mathrm{G}$ protein signaling. Cell. Mol. Neurobiol. 23(3), 305-314 (2003)

41. Konrad, M., Merz, W.E.: Regulation of N-glycosylation. Long term effect of cyclic AMP mediates enhanced synthesis of the dolichol pyrophosphate core oligosaccharide. J. Biol. Chem. 269 (12), 8659-8666 (1994)

42. Konrad, M., Merz, W.E.: Long-term effect of cyclic AMP on Nglycosylation is caused by an increase in the activity of the cisprenyltransferase. Biochem. J. 316(Pt 2), 575-581 (1996)

43. Mills, E.J., et al.: Primary prevention of cardiovascular mortality and events with statin treatments: a network meta-analysis involving more than 65,000 patients. J. Am. Coll. Cardiol. 52(22), 17691781 (2008)
44. Haeuptle, M.A., Hulsmeier, A.J., Hennet, T.: HPLC and mass spectrometry analysis of dolichol-phosphates at the cell culture scale. Anal. Biochem. 396(1), 133-138 (2010)

45. Low, P., et al.: Effects of mevinolin treatment on tissue dolichol and ubiquinone levels in the rat. Biochim. Biophys. Acta 1165(1), 102-109 (1992)

46. Charlton-Menys, V., Durrington, P.N.: Squalene synthase inhibitors: clinical pharmacology and cholesterol-lowering potential. Drugs 67(1), 11-16 (2007)

47. Bergstrom, J.D., et al.: Discovery, biosynthesis, and mechanism of action of the zaragozic acids: potent inhibitors of squalene synthase. Annu. Rev. Microbiol. 49, 607-639 (1995)

48. Baxter, A., et al.: Squalestatin 1, a potent inhibitor of squalene synthase, which lowers serum cholesterol in vivo. J. Biol. Chem. 267(17), 11705-11708 (1992)

49. Bergstrom, J.D., et al.: Zaragozic acids: a family of fungal metabolites that are picomolar competitive inhibitors of squalene synthase. Proc. Natl. Acad. Sci. U. S. A. 90(1), 80-84 (1993)

50. Chugh, A., Ray, A., Gupta, J.B.: Squalene epoxidase as hypocholesterolemic drug target revisited. Prog. Lipid Res. 42(1), 37-50 (2003)

51. Matzno, S., et al.: Inhibition of cholesterol biosynthesis by squalene epoxidase inhibitor avoids apoptotic cell death in L6 myoblasts. J. Lipid Res. 38(8), 1639-1648 (1997)

52. Horie, M., et al:: An inhibitor of squalene epoxidase, NB-598, suppresses the secretion of cholesterol and triacylglycerol and simultaneously reduces apolipoprotein B in HepG2 cells. Biochim. Biophys. Acta 1168(1), 45-51 (1993)

53. Abe, I., et al.: Green tea polyphenols: novel and potent inhibitors of squalene epoxidase. Biochem. Biophys. Res. Commun. 268(3), 767-771 (2000)

54. Wu, A.H., et al.: Effect of 2-month controlled green tea intervention on lipoprotein cholesterol, glucose, and hormonal levels in healthy postmenopausal women. Cancer. Prev. Res. (Phila), (2012) 